\title{
Congenital cardiac anomalies in myelomeningocele patients
}

\author{
Iman Moeini Naghani ${ }^{1}$, Taraneh Hashemi Zonouz ${ }^{1}$, Shima Shahjouei ${ }^{1}$, Amir Azar \\ Homayoun $^{2}$, Farideh Nejat ${ }^{1}$, Mostafa El Khashab ${ }^{3}$
}

${ }^{1}$ Department of Neurosurgery, Children's Hospital Medical Center, Tehran University of Medical Science, Tehran, Iran

${ }^{2}$ Department of Neurosurgery, Sina

Hospital, Tehran University of Medical

Science, Tehran, Iran

${ }^{3}$ Department of Neurosurgery, Hackensack

University Medical Center, New Jersey, US

Corresponding author:

Farideh Nejat

Mailbox: Tehran, F. Nejat, 14155-7854

nejat@sina.tums.ac.ir

Tel: +982166420098

Fax.: + 982166930024

Received: 9 August 2013

Accepted: 9 January 2014

Copyright (C) 2014 by

Academy of Sciences and Arts

of Bosnia and Herzegovina.

E-mail for permission to publish:

amabih@anubih.ba
Objective. Myelomeningocele may be isolated but more frequently is associated with other anomalies. Congenital heart disease occurs with different incidence rate in myelomeningocele which is observed more frequently with skeletal malformations. Methods. This study was undertaken in the Children's Hospital Medical Center between 2010 to 2012 to evaluate 75 myelomeningocele patients for cardiac anomalies, with electrocardiography and echocardiography in addition to clinical examination of the cardiopulmonary system. Demographic information, myelomeningocele location and characteristics, orthopedic deformities, neurological deficits and radiographic findings were studied besides cardiologic assessments. Results. The ages of the patients ranged from 1 day to 4 years. The myelomeningocele locations were lumbosacral, lumbar and sacral area in most cases. Physical examination of the heart was abnormal in 6 children, but echocardiography revealed cardiac anomalies in only two children. Both children were female patients with severe scoliosis, multiple rib deficiencies and associated vertebral anomalies. Conclusion. Congenital heart defects are not very common in MMC patients. Female patients with suspicious clinical examinations for cardiac anomalies and associated rib and vertebral anomalies are advised to be investigated by echocardiography to rule out associated cardiac anomalies.

Keywords: Cardiac abnormality, Myelomeningocele, Screening, Echocardiography.

\section{Introduction}

Myelomeningocele (MMC) is a common type of spina bifida, which is frequently associated with hydrocephalus and Chiarimalformation. Sometimes other anomalies, such as anorectal malformations, skeletal abnormalities involving the spinal column or ribs, genitourinary anomalies and congenital heart diseases, are found with MMC (1). Genetic factors, folate deficiency, radiation and gestational medication exposures are suggested etiological factors (2). The clinical features and outcome of MMC patients depend on the neurological impairment and the severity of concomitant malformations $(3,4)$. Most gross anomalies in MMC patients are detected by prenatal ultrasound evaluation, at birth or soon after that, but anomalies involving the internal organs, such as kidneys, gasterointestinal tract and, rarely, the heart, might be undetected (5).

Congenital heart disease is one of the leading causes of morbidity in childhood. Atrial septum defect (ASD), ventricular 
septum defect (VSD) and tetralogy of Fallot (TOF) are the three most common causes of cardiac defects found in infants with MMC. Apart from ASD, most congenital cardiac anomalies are detected in early childhood (6). Congenital heart defects in MMC patients have been observed more frequently in association with skeletal malformations, including scoliosis, hemivertebrae, multiple rib anomalies and chest wall asymmetry (7-9).

Here we report the cardiological screening of our MMC patients over a short period of time and present the incidence and type of congenital heart defects.

\section{Methods}

This study was undertaken at the Children's Hospital Medical Center, Tehran, between March 2010 and April 2012. As a routine protocol, all patients admitted to the neurosurgical department with diagnosis of MMC are investigated for accompanying intracranial and urogenital anomalies, such as hydrocephalus and neurogenic bladder. According to the findings of the physical examination, further paraclinical assessments might be ordered. In this study, we added a more careful evaluation of the cardiopulmonary system, including electrocardiography (ECG) and echocardiography. Demographic information, MMC location and characteristics, prenatal diagnosis, orthopedic deformities, neurological status and radiographic findings (brain CT or MRI to diagnose ventriculomegaly and Chiari malformation) were recorded beside cardiologic assessments. All patients were enrolled in this study after obtaining informed consent.

\section{Results}

Seventy-five patients ( 41 boys and 34 girls) were consecutively enrolled in the study. The age at admission ranged from 1 day to 4 years (mean age 7 months). The children were the products of uneventful pregnancies from healthy parents. All except for five patients were full-term babies. Preterm babies were born at 27 to 33 weeks of gestational age.

Unfortunately MMCs were diagnosed in only 21 children during prenatal ultrasound evaluations, where all of them were discovered between 22 and 38 weeks of pregnancy. None of the patients with cardiac anomalies were diagnosed during prenatal ultrasound study. The MMC defects were located in the lumbosacral area in $30(40 \%)$, lumbar in 16 (21\%), sacral in $14(18.7 \%)$, thoracolumbar in 7 (9.3\%), cervical in $5(6.7 \%)$ and the thoracic area in 3 (4) patients. All MMCs were cystic lesions except for 7 instances that were rachischisis. Cerebrospinal fluid (CSF) leakage was evident at the time of admission in 10 patients. Normal motor function was noted in 18 patients. Paraplegia was found in 20 patients and distal leg weakness with intact proximal force was observed in $37 \mathrm{pa}$ tients, in whom 2 patients had hand weakness due to symptomatic Chiari type II.

Brain CT scan or MRI was performed in all patients, which confirmed ventriculomegaly in 70 patients and pneumocephalus in 2 patients, who had CSF leakage. Symptomatic hydrocephalus was managed with ventriculoperitoneal shunting in 30 children and endoscopic third ventriculostomy in 5 patients. Repeated attacks of stridor and apnea were observed in one patient who was treated for hydrocephalus and then cervical decompression because of persistence after shunting. According to urological evaluation (radiological, ultrasound and electrophysiological), neurogenic bladder was diagnosed in 64 patients at the time of admission. Anal folds were absent in 43 patients at the time of surgery.

Orthopedic deformities observed at admission time were: clubfoot in 15 patients, hip dislocation in 12 patients, and sever flexion deformity of the hip in 5 patients. 


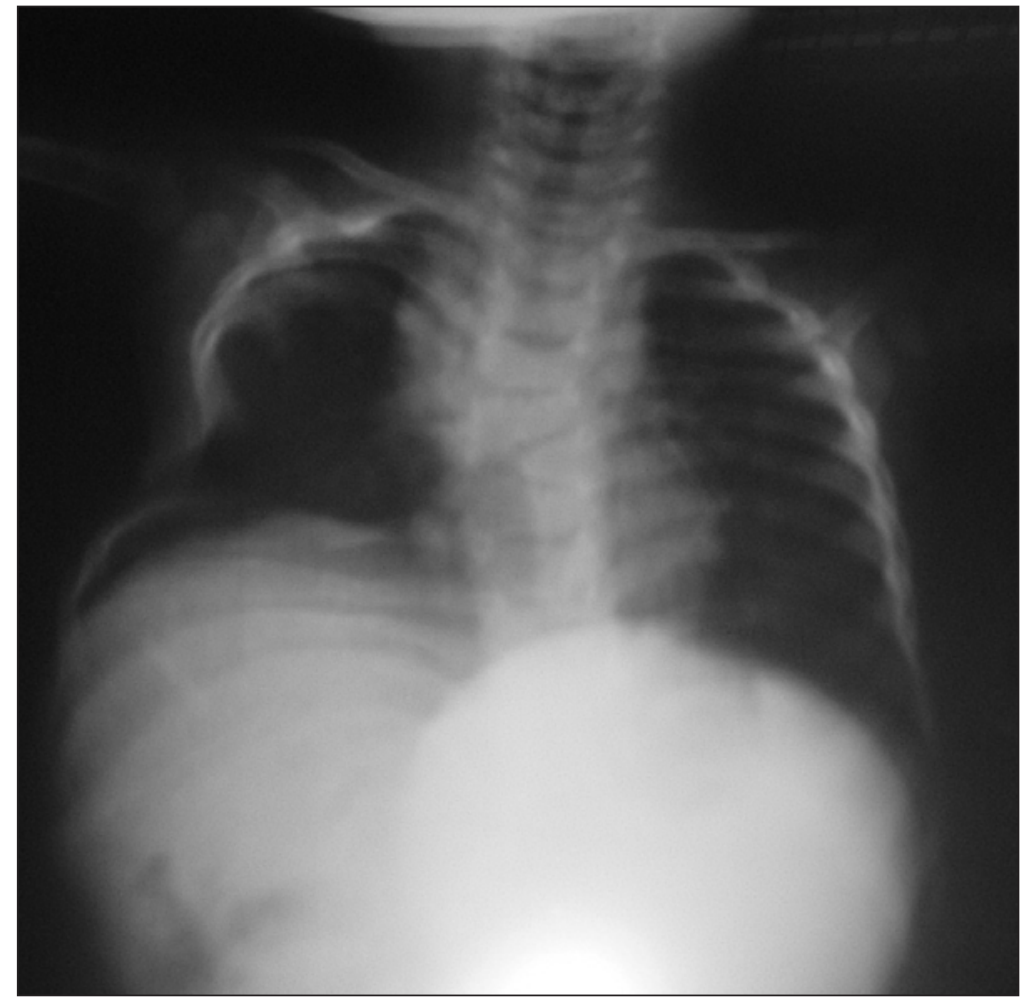

Figure 1 Plain X-ray of patient before surgery shows rib and vertebral anomalies. Notice the soft tissue shadow of the myelomeningocele sac.

Table 1 Associated anomalies in seventy-five patients with myelomeningcele

\begin{tabular}{ll}
\hline Associated anomalies & Number \\
\hline Chiari malformation & 37 \\
Ventriculomegaly & 70 \\
Limb deformity & \\
$\quad$ Clubfoot & 15 \\
$\quad$ Hip dislocation & 12 \\
Neurogenic bladder & 64 \\
Rib anomalies & 5 \\
Cardiac anomalies & 2 \\
\hline
\end{tabular}

Five children had rib anomalies, including absence of one to four ribs found on chest $\mathrm{X}$-ray (Table 1).

Cardiac physical examination was abnormal in 6 children, including abnormal sounds and murmur (systolic Thrill, cyanosis, S3, and harsh systolic murmur) but echocardiography revealed cardiac anomalies in only two of them. One was a 3-month old girl with VSD and ASD. She was admit- ted for management of thoracolumbar MMC and tandem split cord malformations (SCM) type one. She also had scoliosis and multiple rib deficiency. Tetralogy of Fallot was identified in the second patient (Figure 1). She was a 4-month old girl with repeated attacks of cyanosis during crying. She had lumbar MMC and multiple hemivertebra causing scoliosis. A single kidney was noticed in this patient. Both of these children had uneventful neurosurgical operations and are being followed up for management of their cardiac problems.

\section{Discussion}

MMC develops between the 18th and 25th days of gestation, with cord and meninges protrusion through the spinal defect. Association with other congenital anomalies is not uncommon and could happen in the same embryological period or as a conse- 
quence of the underlying disease (1). Genetic and environmental factors have been proposed as the etiology of multiple anomalies in MMC children (10).

Cardiac anomalies associated with MMC have been reported, with diverse incidence rates from several studies ranging between 1.5 to $37 \%(1,11)$. We found an incidence of less than $3 \%$ in this study. The different incidence rates may be related to the diverse methods used for those studies, including the age or gender of the patients. Some cardiac anomalies may be resolved spontaneously as the children grow up, consequently, the incidence of these anomalies could be under-detected when evaluating after the neonatal or infancy period. The degree of detailed cardiological investigations is another factor affecting the chance of detection of some equivocal or small size anomalies.

In concordance with the result of previous studies $(9,11)$, we found that congenital heart anomalies are more frequent in female children with MMC. Cardiac abnormalities have not been reported to occur more often with any specific location of the MMC defect (11). Our study was unable to show any significant relationship between MMC location and heart anomalies either. In this study, skeletal abnormalities involving the ribs and vertebral column (associated with spinal curvature deformity) were found in both MMC children with congenital heart anomalies, while other authors did not mention this association $(1,9-11)$.The detection of cardiac anomalies prior to surgery is important to support the child by giving special care during anesthesia and the operation, and prevent perioperative life threatening complications, such as air emboli or endocarditis.

Clinical examination are able to detect most cardiac anomalies, but some of them may only be detected with echocardiography or ECG. This study could not show a high incidence of cardiac anomalies in
MMC patients, and congenital heart defects were found only in girl infants with gross ribs and vertebral column structural and curvature anomalies.

According to these findings, we do not advise echocardiography routinely in all patients with postnatal diagnosis of MMC. However, any clinical findings of cardiac problem, female sex and associated rib and vertebral anomalies should be considered as risk factors for cardiac anomalies in MMC patients, which necessitate further cardiac investigation by echocardiography, to rule out associated heart anomalies. On the other hand, regarding the importance of prenatal diagnosis of MMC and the associated anomalies, we emphasize routine prenatal ultrasound in any pregnancy to detect these anomalies. In suspicious fetuses for MMC, precise ultrasound evaluation and fetal echocardiography are highly recommended.

\section{Conclusion}

Cardiac abnormalities are not common anomalies in MMC patients. Female patients with suspicious clinical examinations for cardiac anomalies and associated rib and vertebral anomalies should be investigated by echocardiography to rule out associated cardiac anomalies.

Authors' contributions: Conception and design: FN and IMN; Acquisition, analysis and interpretation of data: FN, SS, THZ, IMN and MEK; Drafting the article IMN, THZ and SS; Revising it critically for important intellectual content: SS, MEK and FN.

Conflict of interest: The authors declare that they have no conflict of interest.

\section{References}

1. Baradaran N, Ahmadi H, Nejat F, El Khashab M , Mahdavi A. Nonneural congenital abnormalities concurring with myelomeningocele: report of 17 cases and review of current theories. Pediatr Neurosurg. 2008;44:353-9. 
2. Hamid RKA, Newfield P. Pediatric neuroanesthesia: Neural tube defects. Anesthesiol Clin North America. 2001;19:219-28.

3. Danzer E, Adzick N. Fetal surgery for myelomeningocele: patient selection, perioperative management and outcomes. Fetal Diagn Ther. 2011;30:163-73.

4. Koçak G, Onal C, Koçak A, Karakurt C, Ates O, Cayli SR. Prevalence and outcome of congenital heart disease in patients with neural tube defect. J Child Neurol. 2008;23:526-30.

5. Rai AS, Taylor TK, Smith GH, Cumming RG, Plunkett-Cole M. Congenital abnormalities of the urogenital tract in association with congenital vertebral malformations. J Bone Joint Surg Br. 2002;84(6):891-5.

6. Saifi C, Matsumoto H, Vitale MG, Roye DP Jr, Hyman JE. The incidence of congenital scoliosis in infants with tetralogy of Fallot based on chest radiographs. J Pediatr Orthop B. 2012 Jul;21(4):313-6.

7. Ionescu-Ittu R, Marelli AJ, Mackie AS, Pilote L. Prevalence of severe congenital heart disease after folic acid fortification of grain products: time trend analysis in Quebec, Canada. BMJ. 2009;338:b1673.

8. Botto LD, Olney RS, Erickson JD. Vitamin supplements and the risk for congenital anomalies other than neural tube defects. Am J Med Genet C Semin Med Genet. 2004;125C(1):12-21.

9. Liu YT, Guo LL, Tian Z, Zhu WL, Yu B, Zhang SY, et al. A retrospective study of congenital scoliosis and associated cardiac and intraspinal abnormities in a Chinese population. Eur Spine J. 2011;20(12):2111-4.

10. Hamblet NS, Lijam N, Ruiz-Lozano P, Wang J, Yang Y, Luo Z, et al. Dishevelled 2 is essential for cardiac outflow tract development, somite segmentation and neural tube closure. Development. 2002;129(24):5827-38.

11. Ritter S, Tani LY, Shaddy RE, Minich LL. Are screening echocardiograms warranted for neonates with meningomyelocele? Arch Pediatr Adolesc Med. 1999;153(12):1264-6. 